\title{
Cambios dimensionales de los tejidos en los procedimientos de alargamiento coronario
}

\section{Dimensional changes in tissues after crown lengthening procedures}

\author{
ÁLVAREZ-NOVOA GARCÍA C* \\ BARALLAT SENDAGORTA $L$ * \\ SANZ ALONSO $\mathrm{M} * *$ \\ BASCONES MARTÍNEZ A***
}

\begin{abstract}
Álvarez-Novoa García C, Barallat Sendagorta L, Sanz Alonso M, Bascones Martínez A. Cambios dimensionales de los tejidos en los procedimientos de alargamiento coronario. Av Periodon Implantol. 2012; 24, 2: 103-110.
\end{abstract}

\section{RESUIMEN}

Introducción: Las coronas clínicas cortas son subsidiarias de recibir un tratamiento de alargamiento coronario. En ocasiones es necesario restaurar los dientes después del procedimiento. En estos casos es importante no invadir el espacio biológico para evitar las distintas reacciones del periodonto. El objetivo de esta revisión es analizar los cambios dimensionales que se producen a nivel de los tejidos duros y blandos antes y después de la intervención para poder planificar de forma más precisa la restauración posterior.

Material y métodos: Se realizó una búsqueda en Pubmed con las palabras clave "crown lengthening", limitada a artículos en lengua inglesa, publicados entre los años 1990-2009, que fueran estudios clínicos. La recuperación de los documentos se realizó en la hemeroteca de la Facultad de Odontología de la UCM y en la base de datos Compludoc. Dos evaluadores revisaron los artículos de forma individual.

Resultados y discusión: Los siete artículos, que incluían tanto estudios en animales como en humanos, que fueron incluidos en la revisión, analizaban índice gingival, nivel óseo, profundidad de sondaje, nivel de inserción y anchura biológica, y longitud de la corona y posición de margen gingival.

Conclusiones: El nivel óseo se altera los 3 primeros meses, pero posteriormente se mantiene constante. Los estudios a más largo plazo no encuentran diferencias significativas en profundidad de sondaje a hueso 6 y 12 meses después del alargamiento.

PALABRAS CLAVE: Alargamiento coronario, coronas clínicas cortas, anchura biológica.

\section{SUMIMARY}

Introduction: Short clinical crowns may be treated with crown lengthening techniques. Sometimes teeth need to be restored after the surgery. In these cases it is important not to invade the biological width to avoid the different reactions of the periodontium. The objective of this review is to examine the dimensional changes that occur at the level of hard and soft tissues after surgery in order to plan more accurately the subsequent restoration.

Material and Methods: We performed a search in PubMed with the keyword "crown lengthening", limited to English language articles, that were published between the years 1990-2009, which were clinical trials. The recovery of documents was held in the periodicals of the Faculty of Dentistry in UCM, and in Compludoc database. Two evaluators reviewed the papers individually.

Results and Discussion: The seven articles, including studies in both animals and humans, which were included in the review, analyzing gingival index, bone level, probing depth, attachment level and biological width, and crown length and the position of the gingival margin. 
Conclusions: Bone level is altered during the first 3 months, but thereafter remains constant. The longer-term studies found no significant differences in bone probing depth at 6 and 12 months after the surgery.

KEY WORDS: Crown lenghthening, short clinical crown, biological width.

Fecha de recepción: 1 de marzo de 2010.

Fecha de aceptación: 21 de marzo de 2010.

\section{INTRODUCCIÓN}

Según la Academia Americana de Periodoncia (1992) se define corona clínica como la porción del diente que se extiende desde el borde incisal u oclusal hasta el margen gingival (1). Cuando esta longitud está disminuida, nos encontramos con lo que se conoce como corona clínica corta. Esta alteración puede deberse a defectos del diente (caries, erosión, malformación dentaria...), a traumatismos (fracturas), a causas iatrogénicas (tallados demasiado agresivos, perforaciones durante la endodoncia...) o a una erupción anómala, como por ejemplo la erupción pasiva alterada (Fig. 1)(2). Los dientes con coronas clínicas cortas son candidatos de someterse a un procedimiento de alargamiento coronario ya sea quirúrgico o por medio de un tratamiento ortodóncico. El objetivo que se persigue con el alargamiento coronario quirúrgico es trasladar apicalmente el margen gingival para conseguir elongar la corona clínica y, al mismo tiempo, respetar el espacio biológico de inserción (2). Las técnicas quirúrgicas utilizadas para conseguirlo son : gingivectomía, colgajo de reposición apical (CRA) con cirugía ósea y CRA sin cirugía ósea. Antes de elegir la técnica a realizar es necesario realizar una serie de medicio-

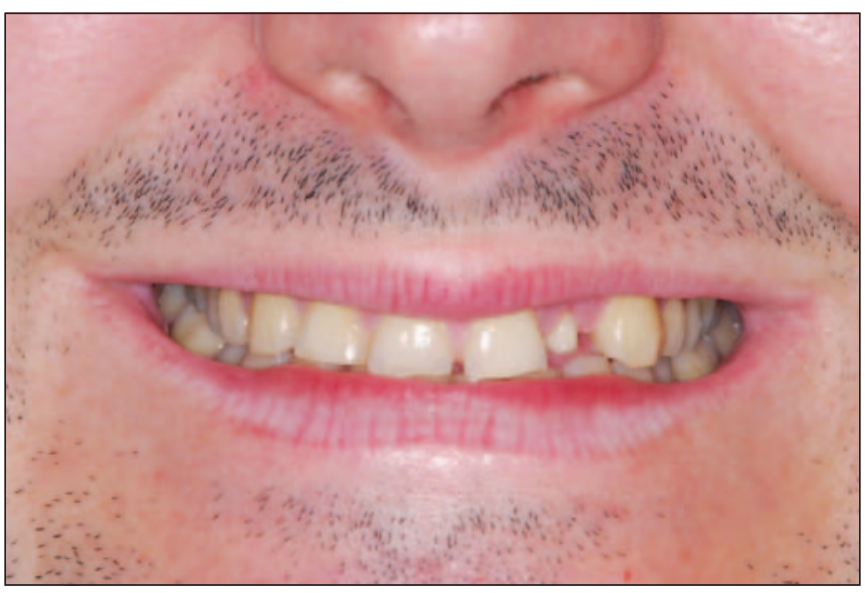

Fig. 1: Corona clínica corta en 22. nes que incluyen: longitud de la corona clínica, longitud de la corona anatómica, anchura de la encía adherida, localización de la cresta ósea, nivel de inserción y grado de inflamación. En los casos de erupción pasiva alterada, en los que habitualmente no se requiere modificar el nivel óseo, el tratamiento indicado consiste en realizar una gingivectomía, o un colgajo de reposición apical sin remodelación ósea. En estos casos generalmente no es necesario restaurar los dientes posteriormente. Sin embargo en los casos en que hay que modificar el nivel óseo, lo habitual es realizar un CRA con cirugía ósea para conseguir una distancia adecuada del margen de la restauración a la cresta alveolar respetando así la anchura biológica (2-4).

En el año 2000, Gunay y cols. demostraron que cuando el margen de la restauración se encontraba invadiendo el espacio biológico, se producía una afectación periodontal a ese nivel. Observaron que el índice gingival y la profundidad de sondaje estaban aumentados en aquellas zonas en las que el margen de la restauración estaba a menos de $1 \mathrm{~mm}$ de distancia de la cresta alveolar (5). En función del individuo esta situación puede producir: inflamación crónica del periodonto, pérdida de hueso alveolar, recesión gingival y/ o formación de una bolsa periodontal (2).

El término de anchura biológica, aunque es familiar para la mayoría de los profesionales, sigue resultando confuso en cuanto a su relevancia clínica. Se define como la dimensión de tejido blando insertada en la porción del diente coronal a la cresta alveolar (Cohen, 1962). Según los estudios sobre cadáveres realizados por Gargiulo y cols. mide entorno a 2,04 mm: 1,07 mm corresponden a las fibras supracrestales del tejido conectivo insertadas en el cemento y $0,97 \mathrm{~mm}$ a la unión epitelial $(2,6,7)$. Sin embargo, hay que tener en cuenta que estas dimensiones son variables en función de la edad del paciente, el tipo de diente y la existencia o no de patología periodontal, sobretodo con respecto a nivel de la unión dentoepitelial (2). De forma clásica, basándose en estudios realizados sobre cadáveres y observaciones empíricas, se ha aceptado que 
la anchura biológica incluyendo la profundidad del surco, es de unos $3 \mathrm{~mm}$. Recientemente Barboza y cols. han realizado un estudio para evaluar las dimensiones de los tejidos blandos supracrestales (incluyendo el surco gingival) en 100 pacientes con periodonto sano mediante sondajes a hueso. Observaron que las medidas variaron entre 1 y $6 \mathrm{~mm}$ siendo de media $3,4 \pm 0,8$ $\mathrm{mm}$ en el caso de las mujeres y $3,2 \pm 0,8 \mathrm{~mm}$ en el caso de los hombres (7).

En aquellos casos en los que el margen de la restauración vaya a quedar muy próximo a la cresta alveolar está indicado realizar un procedimiento de alargamiento coronario (3). Barboza y cols. consideran que, debido a las diferencias encontradas entre sexos y en los distintos grupos dentarios con respecto a las dimensiones de los tejidos blandos supracrestales, a la hora de realizar un alargamiento coronario, habría que individualizar cada caso eliminando la cantidad de tejido necesaria para que se restablezca una anchura biológica adecuada (7).

Sabiendo que una de las metas de la cirugía de alargamiento coronario es conseguir que la restauración ulterior no interfiera con la anchura biológica y sea compatible con un estado de salud periodontal, sería interesante conocer el nivel óseo y la anchura biológica que se restablece tras una cirugía de alargamiento coronario, en comparación con la situación previa (Figs. 2 y 3). El objetivo de esta revisión es analizar los cambios dimensionales que se producen a nivel de los tejidos duros y blandos antes y después de la intervención para poder planificar de forma más precisa la restauración posterior.

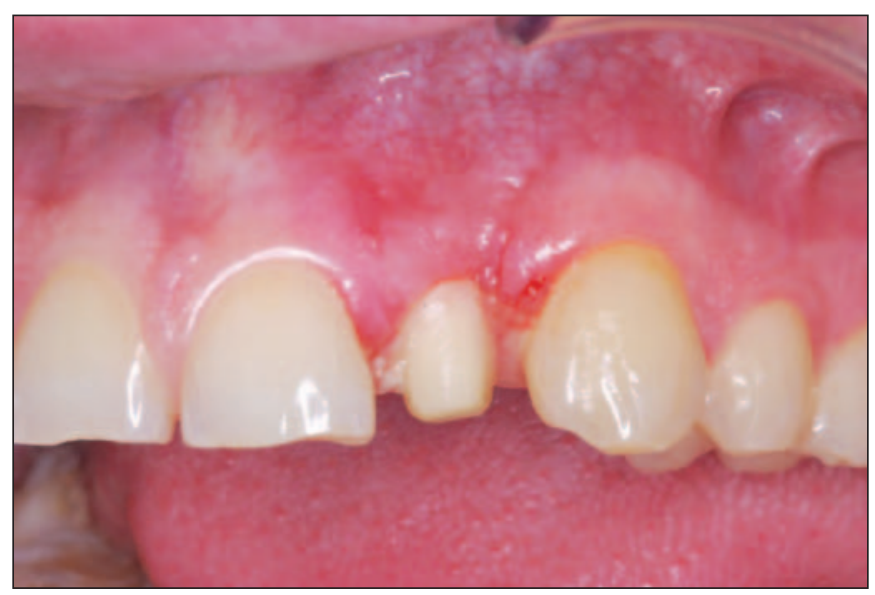

Fig. 2: Pilar una semana después de la cirugía de alargamiento coronario.

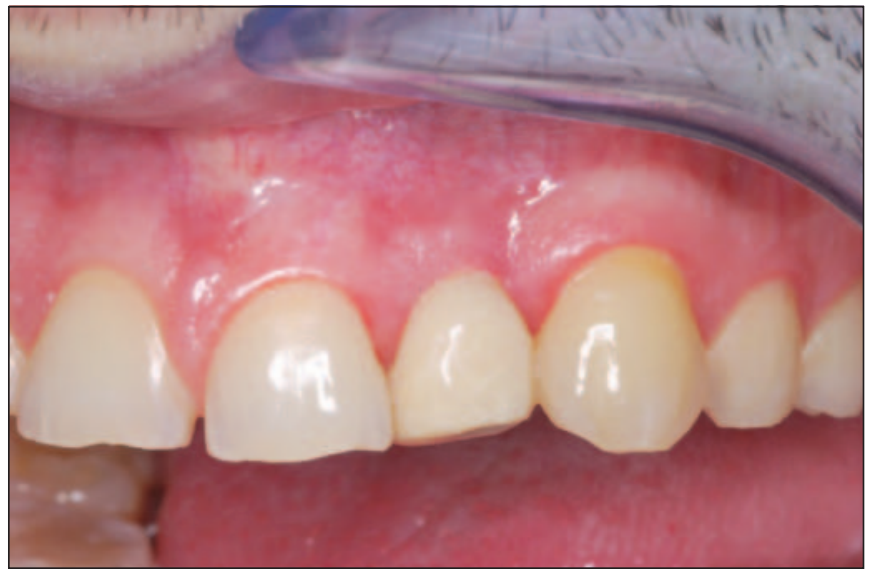

Fig. 3: Restauración provisional de la corona 1 mes después de la cirugía.

\section{MATERIAL Y MÉTODOS}

Inicialmente se realizó una búsqueda on-line a través de pubmed con las palabras clave "crown lengthening", con lo que se encontraron 428 artículos. A continuación, se limitó la búsqueda a artículos publicados en lengua inglesa, comprendidos entre los años 1990 y 2009 y, se descartaron aquellos que fuesen artículos de opinión, casos clínicos y revisiones de la literatura.

Para la selección de los artículos se leyeron los resúmenes y se descartaron todos aquellos que no analizasen los cambios dimensionales en los tejidos duros y blandos, antes y después de la cirugía de alargamiento coronario a través de las siguientes variables: índice de placa, índice gingival, nivel óseo y profundidad de sondaje. Esto nos permite una mayor y mejor comparabilidad entre los estudios.

La recuperación de los documentos se llevó a cabo únicamente en la hemeroteca de la Facultad de Odontología de la Universidad Complutense de Madrid y on-line a través de la base de datos Compludoc.

En definitiva, 7 artículos fueron incluidos en la revisión, todos ellos estudios clínicos. Cinco de estos 7 se obtuvieron mediante la búsqueda avanzada en pubmed, y los 2 restantes se encontraron a través de los "artículos relacionados" propuestos por dicha base de datos.

Dos evaluadores revisaron cada uno de los artículos de forma individual. 


\section{RESULTADOS Y DISCUSIÓN}

De todos los artículos encontrados en nuestra búsqueda sólo cinco de ellos analizan los cambios dimensionales de los tejidos posteriores al procedimiento quirúrgico de alargamiento coronario. Sin embargo, dos más fueron incluidos en la revisión por que a criterio de los revisores, tienen relevancia en el tema estudiado.

\section{Estudios en animales}

Oakley y cols. (1999) realizaron, en Londres, un ensayo clínico en primates con el objetivo de determinar si la anchura biológica se restablece tras la cirugía de alargamiento coronario. Para ello, utilizaron tres monos en los que se seleccionó aleatoriamente un cuadrante y dientes contralaterales como controles. Cuatro o cinco semanas después de la cirugía, la cicatrización, clínicamente, parecía completa. Ocho semanas después se sacrificó a los animales y el estudio histométrico fue realizado por un histólogo independiente que desconocía la existencia del ensayo clínico. En este estudio se observa que los tejidos blandos supracrestales del maxilar superior se reducen tras doce semanas del procedimiento. Siendo la altura del epitelio $0.94 \mathrm{~mm}$ en las zonas tratadas, frente a $1,23 \mathrm{~mm}$ en los controles y la del conectivo $0,63 \mathrm{~mm}$ frente a $1,02 \mathrm{~mm}$ en los controles. Las zonas mandibulares sometidas a cirugía cicatrizaron con un epitelio de unión más largo (1,75 mm frente a 1,53 mm en los controles) Mientras que en el conectivo se produce una reducción en su altura $(0,86$ $\mathrm{mm}$ frente a $0.96 \mathrm{~mm}$ en los controles).

En el maxilar superior con respecto a la reabsorción ósea se observó una reducción media de $0,50 \mathrm{~mm}$ en el maxilar superior y $0,77 \mathrm{~mm}$ en el inferior.

Estos resultados indican que tras la cirugía ósea de alargamiento coronal se produce un epitelio de unión que se extiende hasta el nivel apical del raspado y alisado radicular realizado durante el procedimiento quirúrgico. La reabsorción post quirúrgica de la cresta dejó un espacio para el tejido conectivo supracrestal que alcanzó una altura total inferior a la del lado de control. En definitiva, tras la cirugía se restablece una anchura biológica reducida (8).

A pesar de que los estudios en animales pueden aproximarnos a la respuestas biológicas que tiene lugar en los seres humanos, el número de individuos estudiados es muy bajo.

\section{Estudios en humanos}

Se han encontrado 5 estudios clínicos (Brägger y cols. 1992 (9); Pontoriero y Carnavale 2001 (10); Lanning y cols. 2003 (11); Deas y cols. 2004 (12); Pérez y cols. 2007 (13) cuyo objetivo es analizar los cambios en los tejidos periodontales como resultado de los procedimientos de alargamiento coronario.

Estos estudios realizan una media de 42,4 procedimientos de alargamiento coronario para aumentar la retención de prótesis fija, la accesibilidad a caries o márgenes subgingivales y/o corrección de la estética del sector anterior. Para determinar los cambios dimensionales óseos y gingivales que tienen lugar analizan una serie de variables en baseline, postcirugía y en distintos momentos del seguimiento.

Brägger y cols. 1992, en Berna, evalúan en 25 pacientes ( $28-81$ años), 85 dientes: en 43 realizan alargamientos coronarios y 42 son utilizados como controles. De los 43 dientes tratados, 7 fueron incisivos, 8 caninos, 20 premolares y 8 molares. Para asegurar la reproductibilidad de las mediciones se utiliza una férula. Los parámetros clínicos evaluados en 6 localizaciones (MV,V, $\mathrm{DV}, \mathrm{DL}, \mathrm{L}, \mathrm{ML})$, fueron: índice de placa, índice gingival, nivel de inserción, distancia de la férula al hueso y de la férula al margen. Estos datos se recogen al inicio, 6 semanas y 6 meses después del examen (9).

Pontoriero y Carnavale en 2001 , realiza en 30 pacientes con una media de 40,5 años de edad, 84 intervenciones de alargamiento coronario. Realiza en el diente y la raíz marcas o rieleras como puntos de referencias, para estandarizar las mediciones (índice de placa, índice gingival, posición del margen gingival, profundidad de sondaje, nivel de inserción clínica y nivel óseo), en cuatro localizaciones $(\mathrm{V}, \mathrm{D}, \mathrm{L}, \mathrm{M})$ al inicio del estudio, antes y después de la cirugía, al mes a los 3, 6, 9 y 12 meses (10).

Lanning y cols., en 2003 realizan sobre 23 pacientes con una media de edad de 39 años, intervenciones de alargamiento coronario. Utiliza una férula para realizar las mismas mediciones que en el estudio de Pontoriero pero en distintas localizaciones (MV,DV, $\mathrm{DL}, \mathrm{ML}$ ) al inicio del estudio, durante la cirugía, y al mes, a los 3 y a los 6 meses (11).

Deas y cols. en 2004, realizan 43 cirugías en dientes posteriores de 25 pacientes. Se analizan las mismas variables además de la anchura biológica que en el estudio de Lanning, ayudándose de una férula para realizar las mediciones al inicio, durante la cirugía, al 
mes, a los 3 y a los 6 meses. Evalúa 8 localizaciones en molares, 6 en premolares y 4 en los dientes adyacentes (12).

Pérez y cols. en el año 2007 realizan 19 alargamientos coronarios ( 1 canino, 9 pm y $9 \mathrm{~m}$ ) en los que miden el índice gingival, índice de placa, profundidad de sondaje y nivel óseo, al inicio, durante y a los 6 meses de la cirugía en 6 localizaciones (MV, V, DV, DL, L, ML) utilizando una férula para asegurar la reproductibilidad de las mismas (13).

Vamos a comparar las variables que son comunes a los cinco estudios (índice de placa, índice gingival, nivel óseo y nivel del margen gingival) antes de la cirugía, después, y a los 6 meses. El estudio de Pontoriero hace el seguimiento hasta el año, así que, aunque no podemos comparar sus datos con otros estudios, vamos a reflejar sus resultados por considerarlos de interés.

\section{Índice de placa y gingival}

Ninguno de los estudios encuentra cambios significativos entre los valores de placa y sangrado al sondaje iniciales y a los 6 meses (9-14). El estudio de Pontoriero y Carnavale tiene el mismo resultado para los valores a los 12 meses de la intervención (10).

\section{Nivel óseo}

Los resultados de Brägger y cols. no pueden tenerse en consideración ya que ellos mismos reconocen un error en la forma de realizar sus mediciones (9).

Herrero y cols., realizaron un estudio en el que compararon la dimensión supracrestal postcirugía de alargamiento coronario, realizadas por profesionales con distinto grado de experiencia, con las dimensiones de $3 \mathrm{~mm}$ deseadas previamente. Observaron que por lo general, no se alcanzó una anchura de $3 \mathrm{~mm}$ (de media se redujo entre 1,4 y $2,4 \mathrm{~mm}$ ), que en vestibular es donde más tejido se eliminó y en las localizaciones distolingual y lingual menos (posiblemente por el peor acceso), y que, cuanto más experimentado era el profesional, más se aproximaba a la anchura deseada durante el plan de tratamiento. Los autores concluyen que si el objetivo en las cirugías de alargamiento coronario es conseguir un espacio de $3 \mathrm{~mm}$, los profesionales, deberían de ser más agresivos en la eliminación de tejido (14).
Por su parte Pontoriero y Carnavale observan una reducción media de la cresta alveolar de $0,9 \mathrm{~mm}$ en interproximal y de $1,0 \mathrm{~mm}$ en bucal/lingual y no evalúa los cambios en el nivel óseo en los meses posteriores a la cirugía (10).

Lanning y cols. eliminan en el $90 \%$ de las localizaciones una cantidad igual o mayor de $3 \mathrm{~mm}$ de hueso, y encuentran que los niveles óseos no se mantienen constantes durante los 3 primeros meses posteriores a la intervención, desplazándose apicalmente una media de 0,77 mm en los dientes tratados. No se observan cambios de los 3 a los 6 meses (11).

Deas y cols. miden la cantidad de osteotomía que realizan que resulta ser menor o igual a $1 \mathrm{~mm}$ en el 67,9 de las localizaciones (1,13 $\pm 0,90 \mathrm{~mm}$ de media) (12).

Dos de los estudios (Pérez y cols. y Lanning y cols.) evalúan la validez de la medición del nivel óseo a través del surco gingival, viendo las diferencias que existen entre estas mediciones y las que realizan directamente sobre el hueso tras elevar el colegado. Ambos estudios coinciden al afirmar que ambos métodos son igual de sensibles $(11,13)$ (Figs. 4 y 5 ).

\section{Profundidad de sondaje, nivel de inserción y anchura biológica}

En el estudio de Deas y cols. se demostró que la profundidad de sondaje disminuía de forma estadísticamente significativa si comparaban los valores iniciales, con los valores después de 1, 3 y 6 meses del tratamiento, para cada uno de los grupos. Aunque tien-

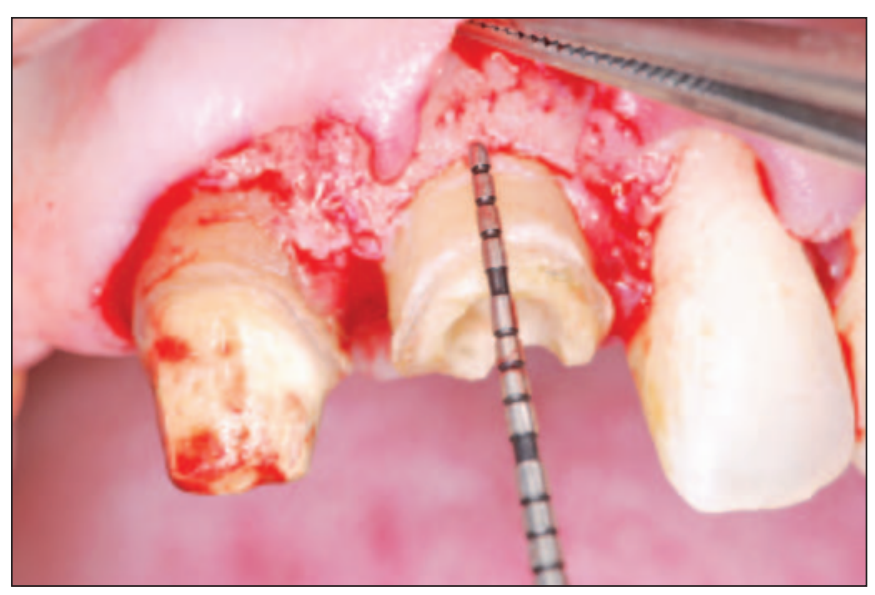

Fig. 4: Nivel óseo antes de la remodelación. 


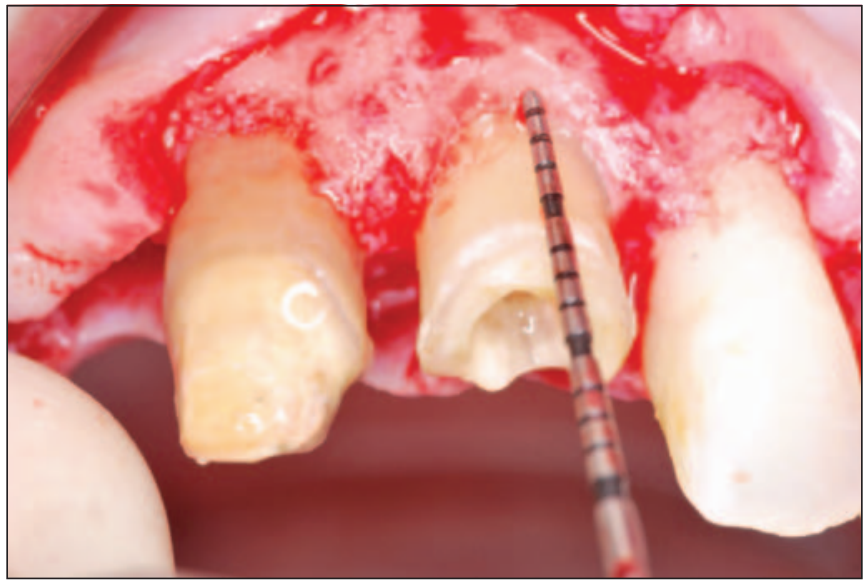

Fig. 5: Nivel óseo después de la remodelación. Se han eliminado 2 mm de hueso.

den a volver a los valores iniciales (12). Sin embargo, $\mathrm{ni}$ el estudio de Lanning ni el de Pontoriero encuentran diferencias estadísticamente significativas a los 6 y 12 meses respectivamente $(10,11)$. Lanning si encuentra diferencia significativa entre los grupos tratados y no tratados adyacentes o no adyacentes a los 3 meses, siendo la profundidad de sondaje en los sitios tratados una media de $3,17 \pm 0,74 \mathrm{~mm}$ mayor que en las localizaciones no tratadas adyacentes y no adyacentes (11).

La anchura biológica es menor a los 3 y 6 meses que antes de realizar el procedimiento de alargamiento coronario en los dientes adyacentes a los dientes tratados, mientras que en las distintas localizaciones del diente tratado en las diferencias no son estadísticamente significativas (11).

Todos los estudios, excepto el de Brägger que no lo evalúa, obtienen una pérdida de inserción clínica estadísticamente significativa al mes, y a los 3 y 6 meses del tratamiento (9-14).

\section{Longitud de la corona y posición del margen gingival}

En los sectores posteriores la media de alargamiento conseguido inmediatamente después de la cirugía es de $2,27 \pm 1,1 \mathrm{~mm}$. El aumento de la longitud de la corona es significativo a los 6 meses de la intervención, sin embargo se observa una tendencia a volver a los valores iniciales (12). Es importante conocer que en los dientes vecinos, tanto en las localizaciones adyacen- tes como no adyacentes a los dientes tratados también se produce un aumento significativo de la corona en todos los momentos del estudio de Deas, en el que se realiza el alargamiento mediante una cirugía con colgajo de reposición apical (12).

El estudio de Pontoriero y Carnavale revela que la cantidad de alargamiento conseguido al año del tratamiento es mayor en las superficies bucales y linguales que en las interproximales $(0,5 \mathrm{~mm}$ en interproximal y de $1,2 \mathrm{~mm}$ en bucal y lingual) (10).

Mientras que Brägger afirma que el margen gingival permanece prácticamente estable a las 6 semanas y 6 meses postquirúrgicos (9), Pontoriero y Carnavale observan una migración coronal del margen de $2,9 \mathrm{~mm}$ en superficies bucales y linguales y de $3,2 \mathrm{~mm}$ en las interproximales, siendo esta diferencia estadísticamente significativa. Además especifican que este crecimiento coronal tiende a ser mayor en biotipos gruesos que en finos (10). En esta línea Deas afirma que la posición en que se sutura el colgajo tiene importancia en la cantidad de tejido que migra coronalmente. Así el crecimiento tisular es mayor cuando el margen gingival se posiciona $1 \mathrm{~mm}$ sobre la cresta ósea que cuando se coloca más coronal, independientemente de la localización (12) (Figs. 6 y 7).

\section{CONCLUSIONES}

Los estudios en animales revelan que tras el procedimiento de alargamiento coronario se restablece una anchura biológica reducida.

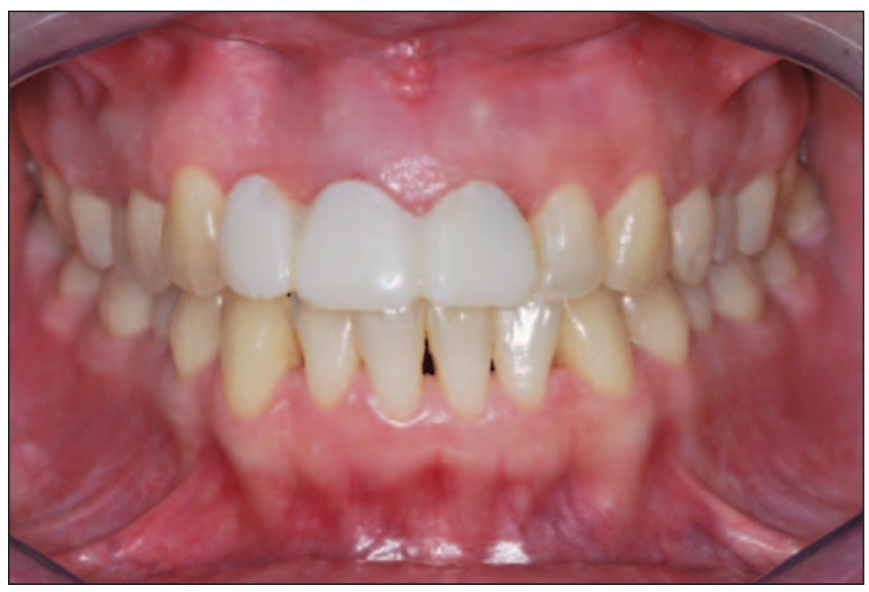

Fig. 6: Situación del paciente antes de la cirugía con una restauración provisional. 


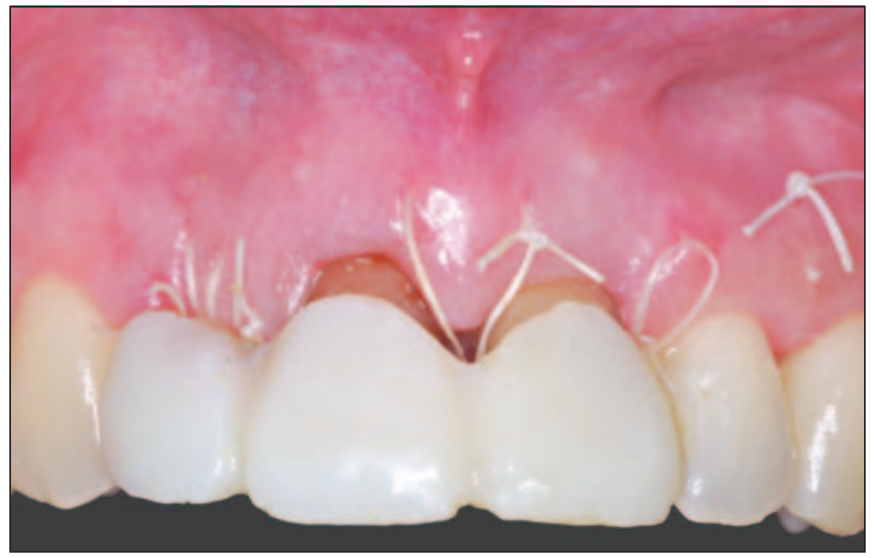

Fig. 7: Posición más apical del margen gingival inmediatamente después de la cirugía.

El nivel óseo en humanos parece alterarse los 3 primeros meses tras la intervención pero posteriormente se mantiene constante.

No hay consenso en si la profundidad de sondaje a hueso antes y después del alargamiento se mantiene constante o si varía. Sin embargo los estudios a más largo plazo no obtienen diferencias estadísticamente significativas a 6 y 12 meses.

El aumento en la longitud de la corona es significativo a los 3, 6 y 12 de la intervención, sin embargo los valores tienden a volver a los iniciales.

Algunos estudios mantienen que la posición del margen gingival permanece estable por lo menos hasta los 6 meses postquirúrgicos, mientras que en otros estudios se observó un crecimiento coronal de los tejidos a los 12 meses, estadísticamente significativo, que parece ser mayor cuando el colgajo se sutura dejando $1 \mathrm{~mm}$ de cresta ósea expuesto.

\section{AGRADECIMIENTOS}

Silvia Oteo Morrilla.

Xavier Costa Berenger.

Simone Fabrizi.

Alberto Ortiz-Vigón Carnicero

\section{BIBLIOGRAFÍA}

1. American Academy of Periodontology. Glossary of periodontal terms. $3^{\mathrm{a}} \mathrm{Ed}$. Chicago 1992:11.
2. Villaverde Ramírez G, Blanco Carrión J, Ramos Barbosa I, Bascones Ilundain J, Bascones Martínez A. Tratamiento quirúrgico de las coronas clínicas cortas: técnicas de alargamiento coronario. Av Periodon Implatol 2000; 12:117-26.

3. Padbury Jr A, Eber R, Wang H-L. Interactions between the gingiva and the margin of restorations. J Clin Periodontol 2003;30:379-85.

4. Goldberg PV, Higginbottom FL, Wilson TG Jr. Periodontal considerations in restorative and implant therapy. Periodontol 2000;25:100-9.

5. Gunay H, Seeger A, Tschernitschek H, Geurtsen W. Placement of the preparation line and periodontal health- a prospective 2- year clinical study. Int J Periodontics Restorative Dent. 2000;20:171-81.

6. Lindhe J,Wennström JL, Berglundh T. La mucosa en áreas dentadas y en áreas periimplantarias. En Lindhe J, Lang NP. Periodontología clínica e implantología odontológica. Editorial médica panamericana. $5^{\mathrm{a}}$ Ed. 2008:6985.

7. Porto Barboza E, Feres Montealto R, Farias Ferreira V, Rocha Carvalho W. Suprecrestal gingival tissue measurements in healthy human periodontum. Int J Periodontics Restorative Dent. 2008;28:55-61.

8. Oakley E, Rhyu IC, Karatzas S, Gandini-Santiago L, Nevins M, Caton J. Formation of the biologic width following crown lengthening in nonhuman primates. Int J Periodontics Restorative Dent. 1999;Dec 19(6):52941.

9. Brägger U. Lauchenauer D and Lang NP: Surgical lengthening of the clinical crown. J Clin Periodontol 1992;19:58-63.

10. Roberto Pontoriero and Gianfranco Carnavale. Surgical Crown Lenghtening: A 12-Month Clinical Wound Healing. J Periodontol 2001;72:841-84

11. Sharon K Lanning, Thomas C. Waldrop, John C Gunsdley and J Gary Maynard. Surgical crown lengthning: Evaluation of the Biological Width. J Periodontol 2003; 74:468-74.

12. David D Deas, Alan J Moritz, Howard T McDonnell, Charles A Powell, Brian Mealey. Osseous Surgery for Crown Lengthening: A 6-Month clinical study. J Periodontol 2004;75:1288-94. 


\section{AVANCES}

Volumen 24 - No 2 - Agosto 2012

13. José R Pérez, Hyman Smukler and Martha E Nunn. Clinical Evaluation of the Spraosseous gingivae befote and alter crown lengthening. J periodontol 2007;78: 1023-30.

14. Herrero F., Scott JB, Maropis PS, Yukna RA. Clinical comparison of desired versus actual amount of surgical crown lengthening. J Periodontol 1995;66:

568-71.

\section{CORRESPONDENCIA}

Carmen Alvarez-Novoa

Correo electrónico: c_alvareznovoa@hotmail.com 